\title{
Análise das publicações dos enfermeiros docentes da Universidade Estadual de Londrina-PR de 1995 a 2006
}

\section{Analysis of publications by the State University of Londrina nursing faculty, from 1995 to 2006}

\author{
Marcela Maria Birolim; Maria do Carmo Lourenço Haddad²; \\ Maria Helena Dantas de Menezes Guariente ${ }^{2}$
}

\begin{abstract}
Resumo
O objetivo desse estudo foi analisar e classificar a direcionalidade temática e as linhas de pesquisa das publicações feitas pelos docentes do curso de enfermagem da Universidade Estadual de LondrinaPR (UEL) no período de 1995 a 2006. Também foram analisados o local e o tempo de formação, a titulação, a área de atuação dos docentes e a classificação dos periódicos. Como critérios balisadores da classificação dos artigos, adotou-se o QUALIS da Coordenação de Aperfeiçoamento de Pessoal de Nível Superior - CAPES. Os dados foram coletados na plataforma do Curriculum Lattes de cada docente, nos quais se levantou o registro de 183 publicações, editadas em 51 periódicos, sendo 56,9\% na temática organizacional, $31,14 \%$ ao segmento assistencial e $12,02 \%$ na temática profissional. Quanto à classificação QUALIS/CAPES, verificou-se que dos 51 veículos de publicação, 33 deles se enquadravam nas categorias de $\mathrm{A}$ a $\mathrm{C}$, sendo a maioria de veiculação nacional. A análise revelou que os docentes do curso de enfermagem da UEL têm o predomínio do perfil investigativo na área organizacional, além disso, ela pode vir a oferecer direções para futuras pesquisas nas temáticas menos exploradas, visando ao fortalecimento das linhas de pesquisa deste grupo profissional.
\end{abstract}

Palavras-chave: Enfermagem. Docentes. Linhas de pesquisa. Publicação.

\begin{abstract}
The objective of this research was to analyze and classify the prevailing themes and ways of research used in publications made in the years of 1995 to 2006 by teachers from the Nursing Department in the State University of Londrina. The place and time of formation, titles, the field of work of the teachers and the classification of the periodicals were also analyzed. Criteria from QUALIS/CAPES - the national program for the qualification of higher education professionals, were used for the classification of the publications. The data was collected from Curricula Lattes where there was a data base of 183 publications edited in 51 periodicals, out of those, $56,9 \%$ were in the theme of organization, $31,14 \%$ in the field of assistance and $12,02 \%$ with the profession theme. As far as classification of the QUALIS/ CAPES is concerned, out of 51 means of publication, 33 of them fit into categories A to C, most of them published at the national level only. The analysis revealed that the predominance of teachers in the nursing department at UEL have an investigative profile in the organizational field and also suggested future researches in less explored fields in order to strengthen this professional group.

Key words: Nursing. Teachers. Research lines. Publication.
\end{abstract}

\footnotetext{
1 Aluna do curso de Mestrado em Saúde Coletiva da Universidade Estadual de Londrina - Londrina-PR. Endereço: Rua Graúna, $\mathrm{n}^{\mathrm{o}}$. 165, bloco 1, apto 302. E-mail: marcelabirolim@hotmail.com

2 Enfermeira; Professora Doutora do Departamento de Enfermagem da Universidade Estadual de Londrina-PR.
} 


\section{Introdução}

A partir da compreensão de estarmos inseridos num contexto rico pelas diversidades pessoais e profissionais, impregnado pelos fatores sócioeducacionais e políticos que regem o país, a pesquisa em Enfermagem é uma atividade que tem possibilitado a reflexão e a transformação da prática profissional (GUARIENTE, 2005).

$\mathrm{Na}$ trajetória dessa profissão, desde a contribuição nightingaleana, já se obtiveram muitas aquisições para o aprimoramento da profissão, mas, diante de novas realidades e inovações científicas e tecnológicas, é necessário que os enfermeiros legitimem o seu fazer produzindo um saber substantivo, que sustente a prática de enfermagem. Portanto, cabe a esses profissionais não só a prática de cuidar e de ensinar a cuidar, mas a prática da pesquisa e da produção científica.

Severino (2002), Demo (2000) e Luckesi (1998) afirmam que a pesquisa é uma atividade intelectual e um trabalho que resulta da relação do homem com o mundo, como um observador, indagador e interventor. Observador, porque percebe os sinais emitidos pela realidade que o cerca, e consegue identificar as situações que fogem do esperado. Indagador porque pergunta a si mesmo porque tal fenômeno ou situação está acontecendo, busca semelhanças e singularidades nessas manifestações. Interventor, porque se sente impelido a dar respostas a essas inquietações. A pesquisa é um labor porque demanda esforço pessoal na busca de informações sobre o fenômeno observado, que toma tempo e esgota. Dependendo do propósito do pesquisador, o trabalho resulta em esclarecimento ou intervenção sobre o problema; dessa forma, a pesquisa é uma atividade que envolve todos os aspectos do ser humano: o intelectual, o emocional, o fisiológico e o interacional.

A produção de conhecimento da enfermagem brasileira vem sendo alvo de estudos e discussões por parte da comunidade científica da área, salientando-se, em todos eles, o importante papel desempenhado pela pós-graduação stricto sensu no desenvolvimento da investigação científica e consequente geração desse conhecimento. De acordo com Gutiérrez et al., (2002), um dos aspectos analisados refere-se à direcionalidade temática dessa produção organizada em áreas ou linhas de pesquisa que refletem a abrangência do corpo de conhecimentos que vem sendo construído ao longo do tempo.

Almeida (1992) alerta para a importância da produção científica ser organizada em torno de linhas de pesquisas e grupos de pesquisadores, de modo que se criem, condições para a pesquisa em enfermagem cumprir uma de suas funções, que é a melhoria das condições de vida da população. Acrescenta-se a isto o fato da pesquisa em enfermagem gerar e validar conhecimentos necessários e úteis à prática da profissão.

No entanto, todo conhecimento produzido necessita e deve ser transmitido a outras pessoas com o objetivo de levá-las a pensar e a perceber tanto as coisas familiares de modo diferente, com as novas informações. E uma das formas de se transmitir os conhecimentos é por meio da divulgação científica: um trabalho de pesquisa seja ele documental, bibliográfico ou de campo, deve ser publicado para que seus resultados sejam reconhecidos no meio científico. Isso vale para qualquer área profissional, inclusive a enfermagem (COSTA; CARVALHO, 2001).

Diante do atual contexto, o presente estudo teve como objetivo analisar a direcionalidade temática e as linhas de pesquisa das publicações feitas pelos docentes-enfermeiros do curso de Enfermagem da Universidade Estadual de Londrina-PR (UEL) no período de 1995 a 2006, com base no esquema categorial proposto por Carvalho (2000) para o agrupamento da produção científica desta classe profissional. Além disso, buscou-se identificar o local, tempo de formação, a titulação, e a área de atuação dos docentes de enfermagem da referida 
instituição e descrever a classificação dos periódicos de acordo com os critérios QUALIS da Coordenação de Aperfeiçoamento de Pessoal de Nível Superior (BRASIL, 2007).

\section{Material e método}

O estudo pertence à categoria descritivoexploratória com abordagem quantitativa. Foram analisados os Currículos Lattes de 46 docentesenfermeiros do curso de enfermagem da UEL, 38 deles estão lotados no Departamento de Enfermagem e 08 no Departamento de Saúde Coletiva. Cabe ressaltar que foram selecionados apenas os professores efetivos no quadro de carreira docente da UEL, não sendo considerados os docentes contratados por tempo determinado.

Na busca de informações por meio dos currículos, foram selecionados os artigos publicados em periódicos que têm por objetivo divulgar o produto de pesquisas junto à comunidade científica..

Para a coleta de dados, foi utilizado um instrumento pré-elaborado contendo dados de identificação do(s) autor(es), local de formação, titulação, área de atuação, título do periódico, local de publicação e classificação do mesmo pelo QUALIS/CAPES, além da área temática e a linha de pesquisa adotada, de acordo com Carvalho (2000).

QUALIS é o resultado do processo de classificação dos veículos utilizados pelos programas de pós-graduação para a divulgação da produção intelectual de docentes e alunos. Tal processo foi concebido pela Coordenação de Aperfeiçoamento de Pessoal de Nível Superior (CAPES) para atender as necessidades específicas do sistema de avaliação, e baseia-se nas informações fornecidas pelos programas e pela Coleta de Dados. Os veículos de divulgação citados pelos programas de pós-graduação são enquadrados em categorias indicativas da qualidade - A, B ou C e do âmbito de circulação dos mesmos: nacional ou internacional. As combinações dessas categorias denotam a importância do veículo utilizado para a publicação, sendo os de circulação internacional os de maior importância.

O Journal Citation Reports (JCR) é adotado como referência base para a classificação dos periódicos de circulação internacional. O índice de impacto a ser utilizado é obtido a partir da mediana dos índices de impacto dos periódicos específicos de cada área. Assim, foi definido que os periódicos com fator de impacto igual ou superior à mediana da área serão classificados como Qualis Internacional A e abaixo da mediana serão classificados como Qualis International B. Serão classificados como Qualis Internacional $\mathrm{C}$ os periódicos catalogados nas bases Medline, International Pharmaceutical Abstracts (IPA), International Nursing Index (INI); Cumulative Index to Nursing \& Allied Health Literature (CINAHAL), SportDiscus, ERIC, Tropical Diseases Bulletin, Sociological Abstracts, Sociological Abstracts and Linguistics and Language Behavior, Planning/Policy \& Development.

O QUALIS/CAPES nacional classifica os periódicos em A, B ou C. São classificados em A ou B quando atendem aos critérios de composição de corpo editorial e de consultores, além da regularidade e periodicidade em que aparecem, sendo diferenciados quando indexados no SCIELO - classificação A, ou no LILACS, EMBASE, EXCERPTA MÈDICA, PSYCLIT, ou que sejam editados por sociedades científicas nacionais representativas da área, obtendo classificação B. Os demais periódicos que não atendam aos referidos critérios são classificados como C (BRASIL, 2007).

Para a classificação das áreas temáticas, seguiu-se a preconização de Carvalho (2000) que apresenta as áreas: profissional, assistencial e organizacional. A área profissional subdivide-se em Fundamentos do Cuidar na Enfermagem, Ética na Saúde e Enfermagem, Tecnologias de Enfermagem, Concepções Teórico Filosóficas de Enfermagem e História da Enfermagem. A área assistencial é composta por tópicos como: O Processo de 
Cuidar em Enfermagem; Cuidar em Enfermagem no Processo Saúde - Doença, e Determinantes de Qualidade de Vida e Saúde - Doença. E a temática organizacional abrange: Políticas e Práticas em Saúde e Enfermagem; Políticas e Práticas de Educação e Enfermagem; Produção em Saúde e Trabalho na Enfermagem; Gerenciamento dos Serviços de Saúde e de Enfermagem e Informação / Comunicação e Enfermagem.

Os tópicos da categoria profissional referem-se às disciplinas, conteúdos teóricos e experiências que compõem a parte substantiva do saber. Já a categoria assistencial refere-se ao que se cogita acerca dquilo que a consciência profissional dá como real objetivado, e também como tudo aquilo que se possa predicar sobre o "saber-fazer" do profissional. $\mathrm{E}$ a organizacional refere-se às disciplinas teóricas e experiências de ciências afins e correlatas / domínio conexo e que servem às aproximações da realidade no âmbito das atividades do conhecimento, tal como interessa à Enfermagem (CARVALHO, 2000).

A classificação foi feita, com base nos títulos dos artigos, o que denotam por vezes, dificuldades na classificação quanto à descrição mais condizente ao teor do tema desenvolvido nas publicações. Aventamos, ainda, a possibilidade de não terem sido todos os trabalhos publicados, devido à falta de atualização de alguns Currículos Lattes.

\section{Resultados e discussão}

Em relação à caracterização dos professores quanto ao local e tempo de formação, titulação, área de atuação, obtiveram-se os seguintes resultados: dentre os 46 docentes incluídos na pesquisa, observou-se um predomínio de enfermeiros formados na UEL (82,6\%); quanto ao tempo de formação e capacitação, verificou-se que $50 \%$ graduaram-se na década de 80 , e $30,5 \%$ graduaram-se na década de 70 , enquanto os cursos de especialização concentraram-se nas décadas de $80(53,6 \%)$ e 90 (31\%). Já em relação a outras titulações, foram identificados que todos os 46 eram portadores do título de mestre e 20 destes com doutorado, e grande parte dessa formação ocorreu a partir do ano 2000.

Os docentes do curso de enfermagem da UEL tiveram a oportunidade de realizarem sua capacitação por meio de dois convênios efetuados com a Universidade de São Paulo - Escola de Enfermagem e Escola de Enfermagem de Ribeirão Preto-SP, nos anos de 1998 e 2003 respectivamente, cursando as disciplinas teóricas em Londrina, o que efetivamente garantiu a capacitação de todo o corpo docente do curso.

Frente a este movimento que resultou na capacitação da totalidade dos professores deste curso, reforçamos o pressuposto de que permitir ao profissional da saúde e agente social que transforma e se transforma no processo de trabalho nas organizações, a oportunidade de possibilidades até então não cogitadas ou tentadas pela atividade investigativa. Com isso, se busca assegurar um movimento dinâmico de aprendizagem reconstrutiva para si, além da produção do saber que vai de encontro às necessidades da sociedade contemporânea (CASTILHO, 2000).

Tambémé notório que a capacitação dos docentes realizadas estava associada às mudanças estratégicas veiculadas pela Reforma Universitária ao redefinir a carreira do magistério a partir da qualificação dos docentes em nível de pós-graduação, fixando o princípio da unidade ensino-pesquisa, propiciando a criação dos primeiros cursos de mestrado em enfermagem no final da década de 70 e início da década de 80 (BARREIRA, 1993). É também nas décadas de 70 e 80 que ocorreu a instalação do Centro de Estudos e Pesquisa em Enfermagem da Associação Brasileira de Enfermagem (CEPEn), e do primeiro doutorado em enfermagem da América Latina, em cooperação com a escola de Enfermagem da Universidade de São Paulo, respectivamente (ANGERAMI, 1993).

Ainda segundo Barreira (1993), é no início da década de 80 que a produção científica de enfermagem torna-se objeto de estudo de várias enfermeiras pesquisadoras, analisada sob 
diferentes perspectivas. Entre outros aspectos salientados nessas análises, ressalta na produção científica de enfermagem, a hegemonia das universidades das Regiões Sudeste e Sul, as quais passaram a desenvolver cursos de pós-graduação em enfermagem. Indiretamente, foi estabelecida a relação com a concentração de renda nessas regiões.

Quanto à área de atuação, verificou-se que $17,4 \%$ dos docentes atuam especificamente na área de saúde coletiva, e 82,6\%, na área de Enfermagem, nas sub-áreas: fundamentos de enfermagem, saúde do adulto, saúde da criança, saúde da mulher, saúde mental, entre outras. Essa áreas perfazem a diversidade de conhecimentos necessária para o ensino de enfermagem na graduação.

Nos currículos analisados, foi verificado o registro de 183 artigos publicados em 51 periódicos, que se enquadram nas áreas temáticas, demonstrados na Figura 1.

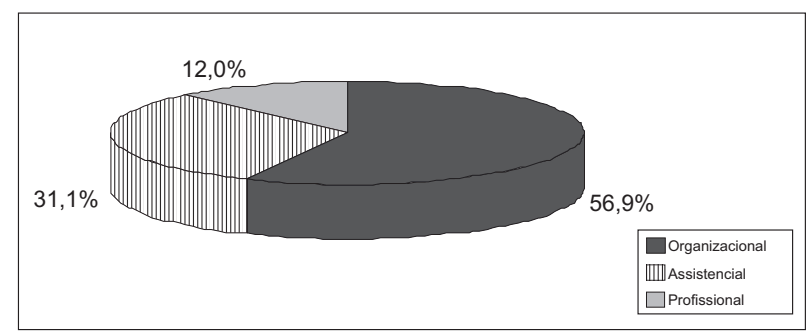

Figura 1. Classificação dos artigos publicados em periódicos por docentes do Curso de Enfermagem da Universidade Estadual de Londrina-PR, segundo área temática, no período de 1995 a 2006.

Os dados mostram um predomínio de temas relacionados à temática organização e administração da assistência e a assistência propriamente dita, confirmando estudo realizado por Lima (2000), entre enfermeiras de serviço. Nesse estudo, a autora verificou que estas duas dimensões do trabalho de enfermagem, a organizacional e a assistencial são vistas como indissociáveis. Acrescenta-se, ainda, a observação feita por Guitierrez et al. (2002) de que o desenvolvimento na enfermagem deve estar comprometido em fazer uma diferença significativa na vida das pessoas a que servimos. Essa está contida nos programas de Pós-Graduação em Enfermagem, que situam a investigação de temas relacionados à área organizacional e assistencial, como reflexo desse compromisso.

Em relação à área profissional, cabe considerar a citação de Almeida (1985, p. 14) de que "ao enfermeiro caberia o papel de reflexão crítica do processo de trabalho da enfermagem, para cumprir o seu papel de intelectual na geração de transformações". O autor reforça a necessidade de mais publicações referentes a essa área.

Guariente (2005), em estudo de doutorado acerca da articulação da atividade investigativa com a prática profissional, argumenta que por meio da pesquisa ganha-se força entre os profissionais e tem-se a possibilidade de criar uma nova cultura em relação à atividade investigativa no contexto profissional. A mesma autora concluiu ainda, que, a partir da instauração da cultura da pesquisa nos serviços de saúde, pode acarretar em um acento positivo mais forte nas relações entre as enfermeiras, seus pares e outros profissionais da equipe de saúde, além de evidenciar o aperfeiçoamento da assistência de enfermagem.

A Tabela 1 mostra que, na temática Organizacional, a linha de pesquisa Políticas e Práticas de Educação e Enfermagem (42,5\%) se sobressaiu em relação às demais linhas de pesquisa desta área. Os artigos publicados nesta linha apresentam títulos estritamente ligados ao campo de conhecimento da Educação. Esta evidência pode associar-se ao fato da reforma curricular ocorrida dentro do curso de Enfermagem da UEL no período selecionado para realização do presente estudo, com o objetivo de aprimorar sua grade curricular visando atender às necessidades da região e aos desejos do corpo docente, por meio da mudança de paradigma na forma de ensinar e aprender enfermagem pela vertente do Currículo Integrado. 
Tabela 1. Linhas de Pesquisa referentes aos artigos publicados em periódicos por docentes do Curso de Enfermagem da Universidade Estadual de Londrina-PR, segundo a área temática Organizacional, no período de 1995 a 2006.

\begin{tabular}{lll}
\hline Linhas de Pesquisa & $\mathbf{N}^{\mathbf{0}}$ & $\mathbf{\%}$ \\
\hline Políticas e práticas de Educação em Enfermagem & 48 & 42,5 \\
Informação/Comunicação em Saúde e Enfermagem & 23 & 20,3 \\
Gerenciamento de serviços de Saúde e Enfermagem & 16 & 14,2 \\
Produção e trabalho em Saúde e Enfermagem & 13 & 11,5 \\
Políticas e práticas em Saúde e Enfermagem & 13 & 11,5 \\
\hline Total & 113 & 100 \\
\hline
\end{tabular}

Soubhia (2004) descreve que o curso de Enfermagem da UEL visa a formar um profissional generalista preparado para atender nos programas de saúde nos níveis primário, secundário e terciário. Esse profissional deve exercer suas funções com consciência, autonomia, compromisso profissional e responsabilidade ética, e contribuir para o desenvolvimento da profissão por meio do ensino, pesquisa, participação nas entidades organizadas e no exercício da cidadania.

A linha Informação / Comunicação e Enfermagem insere produções acerca dos sistemas de informações e os processos de comunicação em saúde e enfermagem. Com o advento das inovações tecnológicas trazidas pelos sistemas de informação e comunicação, houve uma melhora da assistência. Com 20,3\%, a linha Informação / Comunicação apresenta relevância, pois estes meios auxiliam na agilização do processo produtivo, tornando à atenção a saúde mais efetiva e eficaz.

O Gerenciamento dos Serviços de Saúde, outra linha adotada $(14,2 \%)$, traz títulos que se relacionam aos sistemas organizacionais, que são essenciais, pois sustentam teoricamente os serviços de enfermagem e saúde.

Neste sentido, a filosofia e a ciência da administração, seus princípios, teorias, modelos e paradigmas, as novas tecnologias e os processos de gestão de serviços, a organização física e ambiental do trabalho em enfermagem e saúde, as políticas sociais e institucionais de saúde e sua relação com a organização do cuidado, convergem para a construção de conhecimentos ou tecnologias que sustentam as políticas e práticas de gerenciamento dos serviços de saúde e de enfermagem (GUITIERREZ et al., 2002).

As linhas Produção e Trabalho em Saúde e Enfermagem e Políticas e Práticas em Saúde relacionam-se com estratégias de operacionalização de políticas públicas de saúde articuladas à prática da Enfermagem. As políticas públicas de saúde devem ter coerência com as políticas sociais, o compromisso social com a saúde como um direito do cidadão e um dever do Estado. As práticas de enfermagem vêm sendo sustentadas por um saber que se constrói atento às políticas públicas e sociais importantes para a transformação social (GUITIERREZ et al., 2002).

Nos dados apresentados em pesquisa no campo de saúde no Brasil pelo Conselho Nacional de Desenvolvimento Científico e Tecnológico - CNPq (2000) na área temática designada pelo $\mathrm{CNPq}$ como Sistemas e Políticas de Saúde, predominaram os projetos provenientes da área de enfermagem (30 de um total de 53 projetos). Porém, esta área incluía conteúdos de planejamento e gestão de saúde e recursos humanos, bem como sistemas de informação, que no presente estudo foram separados como linhas de pesquisa da área temática organizacional.

A Tabela 2 demonstra que a linha de pesquisa Processo de Cuidar em Enfermagem foi a que congregou maior número de artigos (63,8\%), desenvolvidos na área assistencial, e essa linha 
foi seguida por Determinantes da Qualidade de Vida e Saúde-Doença (19,0\%). Isso demonstra a necessidade de discutir as questões relacionadas às ações de Enfermagem direcionadas ao processo de cuidar / cuidado na promoção, prevenção e recuperação da saúde (que apareceu em minoria), bem como, ao processo saúde-doença e à qualidade de vida de forma integrada.

Tabela 2. Linhas de Pesquisa referente aos artigos publicados em periódicos por docentes do Curso de Enfermagem da Universidade Estadual de Londrina-PR, segundo área temática Assistencial no período de 1995 a 2006.

\begin{tabular}{lll}
\hline Linhas de Pesquisa & $\mathbf{N}^{\circ}$ & $\mathbf{\%}$ \\
\hline Processo de cuidar em Enfermagem & 37 & 63,8 \\
Determinantes da Qualidade de Vida e Saúde-Doença & 11 & 19,0 \\
Cuidar em Enfermagem no Processo Saúde Doença & 10 & 17,2 \\
\hline Total & 58 & 100 \\
\hline
\end{tabular}

De acordo com Dyniewicz (2003) a ação investigativa é fundamental para o cuidar em enfermagem, para que, por meio dela se adquiram, produzam e aprofundem conhecimentos, sem dissociar teoria e prática no cotidiano assistencial da Enfermagem.

Galvão (2002) também ressalta que uma forma de resposta ao desafio de cuidar é a incorporação de conhecimento oriundo da pesquisa na prática profissional, e que compete ao enfermeiro estabelecer com mais precisão sua área de atuação, possibilitando intervenções inovadoras para a solução de antigos e novos problemas, soluções estas promotoras de melhoria nas condições de saúde do paciente.

No entanto, para as demais linhas de pesquisa desta área temática, encontrou-se associação com estudos de perfis epidemiológicos com grupos populacionais e problemas de saúde relevantes, o que responde a mudanças para a melhoria da saúde e qualidade da atenção (GUITIERREZ et al., 2002).

De acordo com o Programa Nacional de Reorientação da Formação Profissional em Saúde da Faculdade de Medicina de Marília, as profundas mudanças políticas, econômicas e sociais ocorridas no mundo todo, na segunda metade do século XX, determinaram uma verdadeira crise de paradigma
(FACULDADE DE MEDICINA DE MARÍLIA, 2005). No Brasil, desde que o Sistema Único de Saúde (SUS) foi legitimado na Lei Orgânica da Saúde, várias propostas de reorientação da assistência à saúde foram-se constituindo no país, numa disputa de modelos de atenção diversos, pautados em diferentes teorias interpretativas do processo saúde-doença. O Programa salienta que:

[...] ainda que o modelo hegemônico pautado na atenção médica, de caráter curativo e hospitalocêntrico seja prevalente, convivemos ao mesmo tempo num modelo que também vem incorporando instrumentos vindos da epidemiologia crítica, do planejamento estratégico em saúde e um projeto ideológico que sustenta a possibilidade de fazermos uma profissão mais comprometida com a realidade social (FACULDADE DE MEDICINA DE MARÍLIA, 2005, p. 18).

$\mathrm{Na}$ área temática profissional (Tabela 3), observou-se que a maioria dos docentes que publicaram trabalhos sobre Fundamentos do Cuidar (75\%) são os mesmos responsáveis por ministrarem esse tema no módulo que aborda o assunto no curso de Enfermagem da UEL, que leva o mesmo título, mostrando a vinculação da prática, do ensino e da pesquisa. 
Tabela 3. Linhas de Pesquisa referente aos artigos publicados em periódicos por docentes do Curso de Enfermagem da Universidade Estadual de Londrina-PR, segundo a área temática Profissional, no período de 1995 a 2006.

\begin{tabular}{lll}
\hline Linhas de Pesquisa & $\mathbf{N}^{\mathbf{0}}$ & $\mathbf{\%}$ \\
\hline Fundamentos do Cuidar na Enfermagem & 15 & 60 \\
Ética na Saúde e Enfermagem & 8 & 32 \\
Tecnologias de Enfermagem & 2 & 8 \\
Concepções Teórico Filosóficas de Enfermagem & 0 & - \\
História da Enfermagem & 0 & 100 \\
\hline Total & 25 & - \\
\hline
\end{tabular}

Echer et al. (1998) contribuem para as reflexões acerca da temática, ao ressaltar que a importância da pesquisa em enfermagem legitima o seu fazer, busca novas formas de cuidar, aproxima as dimensões teóricas e práticas do trabalho de enfermagem, contribui para a qualidade de vida da população, produz o saber substantivo e dá sustentação à prática de enfermagem.

No que se refere à Ética na Saúde e Enfermagem, nota-se um percentual menor de artigos publicados, o que chama a atenção, uma vez que as questões éticas e bioéticas permeiam as práticas de saúde e enfermagem. As inúmeras situações dilemáticas vivenciadas pelos profissionais de saúde em seu ambiente de trabalho, tanto decorrentes dos avanços tecnológicos, de novas concepções ou do próprio modelo de atenção à saúde no Brasil, suscitam um maior número de publicações nesta linha de pesquisa.

Em relação à Tecnologia no Cuidado de Enfermagem, linha de pesquisa encontrada em apenas duas publicações, vale ressaltar a afirmação de Nietsche e Leopardi (2000) em relação a alguns estudos por elas avaliados, da pouca evidência de transposição e transformação desse saber produzido em processos que possam ser considerados tecnologias. Os autores afirmam ainda que para a caracterização de uma profissão é necessária a construção de um corpus tecnológico específico, ou seja, um conjunto de procedimentos transposto em tecnologias.

Rocha et. al. (2008) afirmam ainda que a implementação do cuidado requer a incorporação de processos e produtos e expressões tecnológicas do conhecimento, porém ressaltam que a tecnologia não pode ser vista apenas como algo concreto, como um produto palpável, mas como resultado de um trabalho que envolve um conjunto de ações abstratas ou concretas que apresentam uma finalidade, nesse caso, o cuidado em saúde.

Quanto à classificação QUALIS/CAPES dos periódicos responsáveis pelas publicações em evidência, verificou-se que dos 51 veículos de publicação descritos, 33 estão classificados nesse sistema; e que, dos 183 artigos analisados, 138 foram publicados em periódicos classificados pela QUALIS/CAPES nas categorias A, B ou C em âmbito de circulação nacional ou internacional. O referenciamento desta classificação permitiu verificar que 11 artigos (8\%) foram publicados em periódicos com conceito A, sete publicados em periódicos de circulação internacional e quatro de circulação nacional; 84 (60,9\%) publicados em periódicos com conceito $\mathrm{B}, 25$ de circulação internacional e 59 de circulação nacional; e 43 $(31,1 \%)$ em periódicos com conceituação $\mathrm{C}$, sendo 11 de circulação internacional e 32 de circulação nacional. Nota-se um predomínio de publicações em periódicos de circulação nacional com conceito B (42,8\%).

A importância de um periódico científico deve-se ao seu objetivo de divulgar o produto de pesquisas à comunidade científica e ser a última etapa do processo de produção acadêmica. Um periódico científico remete-nos à importância do ato de escrever, e a linguagem escrita é uma das mais 
relevantes conquistas da humanidade, surgindo como uma forma de socialização do conhecimento (JESUS et al., 2005).

No que tange à quantidade de publicações e a qualidade dos veículos de divulgação, Guariente (2005) aponta sobre as dificuldades pessoais e operacionais dos autores no cumprimento das exigências das revistas, bem como o tempo do aceite e publicação entre outras situações que não têm favorecido os professores e enfermeiros no tocante à disseminação do conhecimento em periódicos considerados de primeira linha.

Vale ressaltar que, dentre os currículos analisados, apenas 26,1\% dos docentes estavam com seus currículos atualizados na plataforma Lattes até 2007, 52,2\% estavam atualizados até 2006 e 21,7\% estavam atualizados a período anterior a 2006. Essa situação sugere um provável aumento no número de publicações, ainda não registradas, em uma nova classificação.

\section{Conclusão}

A relevância em se definir linhas de pesquisa em enfermagem prende-se às exigências atuais do processo de produção do conhecimento, que se tornou um empreendimento coletivo, realizado de modo progressivo e contínuo ao longo do tempo para legitimar a profissão.

Uma linha de pesquisa passa a ter existência como resultado da produção de investigações, que devem atender a certas condições peculiares. As linhas de pesquisa empregadas neste estudo seguiram o esquema categorial proposto por Carvalho (2000), e às publicações realizadas no período de 1995 a 2006, pelos docentes do curso de enfermagem da UEL contaram com uma diversidade de temas, relacionadas às linhas de pesquisa que compõe o referido esquema, em maior ou menor extensão.

As linhas de pesquisa organizacional obtiveram maior quantidade de publicações $(56,9 \%)$, em especial na parte educacional, demonstrando que os docentes do curso de enfermagem da UEL constroem os conhecimentos orientados pela interdisciplinaridade, dada a proximidade com os conteúdos das áreas correlatas, associadas a outras categorias também necessárias para a formação do conhecimento.

A temática assistencial mostrou a preocupação relacionada à promoção da saúde, prevenção e tratamento de doenças e determinantes da qualidade de vida, importantes para o conhecimento e implemento da assistência.

Considerando a complexidade dos problemas relacionados ao campo da saúde e da educação, verificou-se, na área profissional, a necessidade de estudos e pesquisas relativas a concepções teóricas filosóficas de saúde e enfermagem e à história de enfermagem. Também cabe explorar a ética na saúde e a tecnologia em saúde e enfermagem, que também foi pouco explorada pelos docentes.

Um aspecto importante a ser considerado nos resultados desta classificação é relativo às certas dificuldades encontradas no processo de análise dos títulos para incluí-los nas linhas de pesquisa abordadas. Porém, este esquema categorial como instrumento metodológico mostrou-se pertinente e suficiente para alcançar os objetivos propostos.

Espera-se que este estudo contribua para o aprofundamento dos conhecimentos relativos às publicações feitas pelos docentes da UEL, enfatizando as principais linhas de pesquisa adotadas, para que numa visão mais ampliada fortaleça o conhecimento e a compreensão dos fenômenos da profissão, e das ciências afins. Também cabe aplicar esses conhecimentos no ensino e formação dos futuros profissionais e incutir nesta formação a importância da pesquisa e do saber científico para a construção de um corpo de conhecimento próprio que sustente e transforme o ensino e a prática da profissão de enfermagem. 


\section{Referências}

ALMEIDA, M. C. P. Pesquisa, poder e saúde. Revista da Escola de Enfermagem da USP, São Paulo, v. 26, p. 157165, out. 1992. Edição Especial.

ALMEIDA, M.C.P. A prática da enfermagem como subsídio para a formação enfermeiro. In: A PRÁTICA DA ENFERMAGEMEOCURRÍCULO DE GRADUAÇÃO, 1985, Ribeirão Preto. Anais... Ribeirão Preto: Escola de Enfermagem de Ribeirão Preto, Universidade de São Paulo, 1985. p. 53-59.

ANGERAMI, L. S. O desenvolvimento da pesquisa no Brasil.RevistaLatino-americanade Enfermagem, Ribeirão Preto, v. 1, p. 43-52, dez. 1993. Edição Especial.

BARREIRA, I. A. A pesquisa em enfermagem no Brasil e sua posição em agência federal de fomento. Revista Latino-Americana de Enfermagem, Ribeirão Preto, v. 1, n. 1, p. 51-57, jan. 1993.

BRASIL. Ministério da Educação. Coordenação de Aperfeiçoamento de Pessoal de Nível Superior. Qualis. Disponível em: <http://www.capes.gov.br/avaliacao/ webqualis.html>. Acesso em: 2 mar. 2007.

CARVALHO, V. Linhas de pesquisa e prioridades de enfermagem: proposta com distinção gnoseológica para o agrupamento da produção científica de pós-graduação em enfermagem. Mímeo. Documento apresentado no Fórum Nacional de Coordenadores de Programa de Pós-Graduação em Enfermagem, realizado durante o XVI Enf. Nordeste e II Encontro de Pesquisa da RENE. Salvador, Bahia, junho de 2000.

CASTILHO, V. Educação continuada em enfermagem: a pesquisa como possibilidade de desenvolvimento de pessoal. O Mundo da Saúde, São Paulo, v. 24, n. 5, p. 357-360, set./out. 2000.

CONSELHO NACIONAL DE DESENVOLVIMENTO CIENTÍFICO E TECNOLÓGICO - CNPq. Apreciação geral e estratégica da pesquisa no campo de saúde no Brasil. Brasília, DF, 2000.

COSTA, R. S.; CARVALHO, D. V. Análise da produção científica dos enfermeiros de Minas Gerais publicada em periódicos de enfermagem. Revista Latino-americana de Enfermagem, Ribeirão Preto, v. 9 n. 5, p. 19-25, set. 2001.

DEMO, P. Pesquisa: princípio cientifico e educativo. 7. ed. São Paulo: Cortez, 2000.

DYNIEWICZ, A. M. Metodologia da pesquisa para enfermeiros: práticas educativas em hospital universitário. 2003. Tese (Doutorado) - Escola Paulista de Medicina, Universidade Federal de São Paulo, São Paulo.

ECHER, I. C.; CROSSETTI, M. G. O.; PASKULIN, L. M. G.; COGO, A. L. P.; SANTOS, V. B. D.; SOTTOMAIOR, V. S.; TAFFE, R. E.; VICENTE, M. C. Criação da comissão de pesquisa do grupo de enfermagem do
Hospital de Clínicas de Porto Alegre. Revista Gaúcha de Enfermagem, Porto Alegre, v. 9, n. 1, p. 56-59, jan. 1998.

FACULDADE DE MEDICINA DE MARÍLIA. Programa Nacional de Reorientação da Formação Profissional em Saúde. Famema em novas perspectivas: curso de enfermagem. 2005. Disponível em: <htpp:// www.famema.br/documentos/Prosaude_Enfermagem Famema.pdf>. Acesso em: 4 jul. 2006.

GALVÃO, C. M. A prática baseada em evidências: uma contribuição para a melhora da assistência de enfermagem perioperatória. 2002. Tese (Livre Docência) - Escola de Enfermagem de Ribeirão Preto, Universidade de São Paulo, Ribeirão Preto.

GUARIENTE, M. H. D. M. Articulação da atividade investigativa com a prática profissional: processo $e$ produto de enfermeiras apoiadas por um núcleo de pesquisa. 2005. Tese (Doutorado em Enfermagem) Escola de Enfermagem de Ribeirão Preto, Universidade de São Paulo, Ribeirão Preto.

GUITIERREZ, M. G. R. et al. Os múltiplos problemas pesquisados e a pesquisar na enfermagem. Revista Brasileira de Enfermagem, Brasilia, v. 55, n. 5, p. 535541, set./out. 2002.

JESUS, D. S. S. et al. A Revista Mineira de Enfermagem e a Divulgação da Produção Científica na Enfermagem. Revista Mineira de Enfermagem, Belo Horizonte, v. 9, n. 2, p. 147-151, abr.jun. 2005.

LIMA, M. A. D. Concepções de enfermeiras sobre seu trabalho no modelo clínico de atenção à saúde. Revista Brasileira de Enfermagem, Brasília, v. 53, n. 3, p. 343354, 2000.

LUCKESI, C.; BARRETO, E.; COSMA, J.; BAPTIS, N. Fazer universidade: uma proposta metodológica. São Paulo: Cortez, 1998.

NIETSCHE, A. E., LEOPARDI, M. T. O saber de enfermagem como tecnologia: produção de enfermeiros brasileiros. Texto \& Contexto-Enfermagem, Florianópolis, v. 9, n. 1, p. 129-152, 2000.

ROCHA, P. K.; PRADO, M. L.; WAL, M. L.; CARRARO, T. E. Cuidado e tecnologia: aproximações através do modelo de cuidado. Revista Brasileira de Enfermagem, Brasília, v.61, n.1, p. 113-116, 2008.

SEVERINO, A. J. Metodologia do trabalho cientifico. 21. ed. São Paulo: Cortez, 2002.

SOUBHIA, Z. Construção de uma proposta de ensino e aprendizagem de pesquisa em um currículo integrado de enfermagem mediante a comparação de desempenhos, em pesquisa, de graduandos em dois projetos urriculares. 2004. Tese (Doutorado em Enfermagem) - Escola de Enfermagem de Ribeirão Preto, Universidade de São Paulo, Ribeirão Preto. 